\title{
Linear endobronchial ultrasonography: a novelty turned necessity for mediastinal nodal assessment
}

\author{
Robert C Rintoul, Rawya Ahmed, Brendan Dougherty, Nicholas R Carroll
}

Department of Thoracic Oncology, Papworth Hospital, Cambridge, UK

\section{Correspondence to} Dr Robert C Rintoul, Department of Thoracic Oncology, Papworth Hospital, Cambridge CB23 3RE, UK robert.rintoul@nhs.net

Received 29 April 2014 Revised 15 August 2014 Accepted 1 September 2014 Published Online First 22 September 2014

\section{CrossMark}

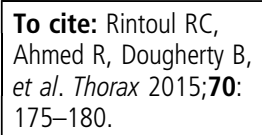

\section{ABSTRACT}

Linear endobronchial ultrasound was first described in 2003. Since then the technique has spread rapidly and has now become an established practice in many centres as the first-line mediastinal investigation for the diagnosis and staging of lung cancer. In combination with endoscopic ultrasound, the majority of the mediastinum can be assessed and this approach has been shown to have equivalent accuracy to surgical staging. This strategy is also cost-effective. New tissue processing techniques using liquid-based thin-layer cytology and cell blocks have increased diagnostic yield using immunohistochemical staining and molecular diagnostics. Several meta-analyses of case series and, more recently, randomised controlled trials have provided high-level evidence of efficacy leading to incorporation into national lung cancer staging guidelines. In addition, linear endobronchial ultrasound is increasingly used in the investigation of mediastinal lymphadenopathy for suspected sarcoidosis, tuberculosis and lymphoma. While undoubtedly endobronchial/endoscopic ultrasound has reduced the need for surgical staging in lung cancer, the latter still has an important role to play in certain scenarios. The challenge now facing clinicians is to learn to apply the appropriate test or sequence of tests in each patient while ensuring that operators are appropriately trained in order to ensure optimal outcomes.

\section{INTRODUCTION}

The first description of endobronchial ultrasound (EBUS) used a mechanical radial mini-ultrasound probe with a single-element transducer contained within a polyethylene sheath passed through the working channel of a flexible bronchoscope. ${ }^{1}$ Radial probe EBUS at a frequency of $20 \mathrm{MHz}$ provides a $360^{\circ}$ image of the airway wall and adjacent structures. Prior to the advent of linear EBUS, some groups used this technique to identify the position of hilar and mediastinal lymph nodes prior to performing non-ultrasound-guided transbronchial needle aspiration (TBNA). ${ }^{2-4}$ Currently, the main role for radial EBUS is for localisation and biopsy of peripheral lung nodules. ${ }^{5}$

The first report of clinical application of linear probe EBUS was published in Thorax in 2003. ${ }^{6}$ In the ensuing decade, the technique has progressed from being a research tool to gaining widespread acceptance and entering routine clinical practice, primarily for the diagnosis and staging of lung cancer. Linear EBUS uses an electronic curved linear array ultrasound transducer mounted at the distal tip of a flexible bronchoscope, which can be covered with a water-inflatable balloon sheath. Several models are available, all of which permit simultaneous viewing of endobronchial and ultrasound imaging at between 5 and $10 \mathrm{MHz}$ allowing tissue penetration up to $50 \mathrm{~mm}$. Real-time TBNA is performed using 21,22 or 25 gauge needles through a $2 \mathrm{~mm}$ instrument channel. The technique for identification and sampling of hilar and mediastinal lymph nodes has been described previously. ${ }^{7}$ EBUS allows access to mediastinal lymph nodes $2 \mathrm{R}$, 2L, 3P, 4R, 4L, 7 and hilar nodes 10R, 10L, 11R and $11 \mathrm{~L}$.

The mainstay of surgical mediastinal staging has been cervical mediastinoscopy and, more recently, video-mediastinoscopy. Mediastinoscopy provides good access to stations 2 and 4 and the upper anterior part of station 7. Access to lymph node stations 5 and 6 , which cannot be routinely accessed by either cervical mediastinoscopy or EBUS, is best provided by left anterior mediastinotomy or video-assisted thoracoscopy (VATS). Newer surgical techniques such as video-assisted lymphadenectomy and transcervical extended mediastinal lymphadenectomy (TEMLA) offering complete lymph node dissection with reported high sensitivity and negative predictive value have been described but are restricted to a few specialist centres.

\section{INDICATION FOR LINEAR EBUS}

The main indications for linear EBUS with TBNA are

A. diagnosis and staging of lung cancer

B. restaging of the mediastinum following neoadjuvant chemotherapy

C. diagnosis of metastatic extra-thoracic malignancy

D. investigation of isolated mediastinal lymphadenopathy
1. sarcoidosis
2. tuberculosis
3. suspected lymphoma.

\section{Diagnosis and staging of lung cancer}

The first clinical studies demonstrating the utility of linear EBUS for diagnosing lung cancer were published as cases series between 2003 and $2006 .^{68-10}$ In 2009, a meta-analysis reported pooled sensitivity for linear EBUS-TBNA of 0.93 (95\% CI 0.91 to 0.94).$^{11}$ More recently, Dong et $a l^{12}$ reported a meta-analysis of nine studies (eight prospective), incorporating 1066 patients that had used EBUS-TBNA as the sole diagnostic method and for which there was surgical confirmation of results. In this updated review, pooled sensitivity was similar at 0.90 (95\% CI 0.84 to 0.96 ) and pooled accuracy 0.96. In the recent American College of Chest Physicians (ACCP) 2013 guidelines, ${ }^{13}$ an updated systematic review by Silvestri et al incorporating 2756 patients revealed an overall median sensitivity 
of $89 \%$ (range $46 \%-97 \%$ ). The median negative predictive value in this review was $91 \%$ with an estimated disease prevalence of 58\%. For cervical mediastinoscopy, the ACCP 2013 review included 9267 patients with a sensitivity of $78 \%$ for detecting $\mathrm{N} 2 / 3$ disease. The sensitivity for videomediastinoscopy was reported as $89 \%$ in 995 patients. The first prospective controlled trial comparing EBUS-TBNA with (video-assisted) mediastinoscopy for mediastinal lymph node staging was published by Yasufuku et al. ${ }^{14}$ In this study, EBUS was performed under general anaesthesia immediately prior to mediastinoscopy. The sensitivity, negative predictive value and diagnostic accuracy for EBUS-TBNA were 81\%, 91\% and 93\%, respectively, and for mediastinoscopy $79 \%, 90 \%$ and $93 \%$. No significant differences were found between EBUS-TBNA and mediastinoscopy in determining $\mathrm{N}$ stage. The authors concluded that EBUS-TBNA might largely replace mediastinoscopy in patients with potentially resectable non-small cell lung cancer given similar efficacy and a lower complication rate.

EBUS-TBNA can also be used to evaluate peri-tracheobronchial intrapulmonary lung masses. Three groups have reported their experience of using EBUS-TBNA to diagnose intrapulmonary masses lying adjacent to a major airway. ${ }^{15-17}$ In these series, diagnostic sensitivity ranged between $82 \%$ and $94 \%$. Technically biopsy of such lesions can be more challenging as a lung mass may be composed of areas of tumour tissue along with areas of inflammation or fibrosis. Therefore, tissue sampling may have to be more extensive to maximise chances of a true positive or negative result. Pneumothorax has been reported as a complication in this setting but seems to be $<1 \%$.

\section{Restaging of the mediastinum following neoadjuvant chemotherapy}

Restaging of the mediastinum following neoadjuvant chemotherapy is challenging and traditionally has been undertaken using mediastinoscopy. It is recognised that re-mediastinoscopy is technically more difficult to perform on account of adhesions and fibrotic change induced by the initial procedure and induction treatment. Several re-mediastinoscopy case series involving small numbers of patients have been reported with sensitivities for detection of residual disease ranging from $29 \%$ to $74 \% .^{18-20}$ In most cases, the 'gold-standard' applied has been analysis of mediastinal nodes removed at thoracotomy, although this can only address the ipsilateral side.

Few studies using EBUS-TBNA have addressed restaging to date. Herth et $a l^{21}$ undertook a retrospective review of 124 patients with tissue-proven stage IIIA N2 disease who had been treated with induction chemotherapy and who had undergone restaging using EBUS-TBNA. Surgical verification was performed in all cases. The prevalence of persistent mediastinal nodal disease was 94\%. Sensitivity for detection of malignancy was $76 \%$ in this series, but the negative predictive value was only $20 \%$. Most recently, Szlubowski et $a l^{22}$ reported a prospective series of 106 patients undergoing combined endobronchial and endoscopic ultrasound (EUS) for induction therapy for non-small cell lung cancer. All patients with negative or uncertain results underwent TEMLA for confirmation. The prevalence of persistent mediastinal nodal disease was 52\%. Overall, diagnostic sensitivity of endosonography for mediastinal metastases was $67 \%$ (95\% CI $53 \%$ to $79 \%$ ), specificity 96\% (95\% CI 86\% to 99\%) and overall accuracy 81\% (95\% CI $73 \%$ to $87 \%)$. The negative predictive value was $73 \%(95 \% \mathrm{CI}$ $61 \%$ to $83 \%)$.

Taken together these studies suggest that initial staging should be performed using EBUS and/or EUS avoiding surgical staging whenever possible. Initial restaging can also be performed using EBUS and/or EUS. However, given the low negative predictive value of EBUS/EUS in the neoadjuvant chemotherapy setting, negative findings should be confirmed by surgical staging (mediastinoscopy) prior to thoracotomy.

\section{Diagnosis of metastatic extra-thoracic malignancy}

Mediastinal lymphadenopathy in patients with a known extrathoracic malignancy is a commonly encountered problem, and EBUS has been shown to play a useful role in determining aetiology. In a multicentre retrospective series of 161 patients, Navani et $a l^{23}$ demonstrated that EBUS-TBNA diagnosed mediastinal or hilar metastases in $71(44 \%)$ of patients, new lung cancer in $20(12 \%)$ and sarcoidosis or a sarcoid-like reaction in 14 (9\%). The final diagnosis prevalence for malignancy was $68 \%$. Using EBUS, the sensitivity, accuracy and negative predictive values for malignancy were $87 \%, 88 \%$ and $73 \%$, respectively. Tournoy et $a l^{24}$ also reported a sensitivity of $85 \%$ for EBUS-TBNA of thoracic lymphadenopathy in 92 patients with extra-thoracic malignancy.

\section{Investigation of isolated mediastinal lymphadenopathy}

Identification of isolated mediastinal lymphadenopathy by CT is a common clinical scenario, and prior to the advent of EBUS it was normally investigated using a thoracic surgical approach, most commonly cervical mediastinoscopy or VATS. The role of EBUS for investigation of isolated mediastinal lymphadenopathy has been described by several groups, but for simplicity it is reviewed here by disease category.

\section{Investigation of suspected sarcoidosis}

Historically, the standard bronchoscopic investigation for patients suspected of having sarcoidosis has been flexible bronchoscopy with endobronchial and transbronchial lung biopsies. Since 2007, several groups have reported their experience of using EBUS-TBNA for assisting the diagnosis of sarcoidosis. A recent systematic review and meta-analysis by Agarwal et $a l^{25}$ identified 15 good quality, predominantly prospective studies involving 553 patients. The diagnostic yield of EBUS-TBNA ranged from $54 \%$ to $93 \%$ with pooled diagnostic accuracy of $79 \%$ (95\% CI 71\% to 86\%). Diagnostic yield was noted to be significantly higher in the prospective studies compared with retrospective studies. Of note, the diagnostic yield of EBUS-TBNA surpassed that of traditional bronchoscopic biopsy techniques. Combining EBUS-TBNA with transbronchial lung biopsy and/or endobronchial biopsy should increase diagnostic accuracy yet further. The first randomised controlled trial to compare the diagnostic accuracy of EBUS-TBNA with transbronchial biopsy in patients with suspected sarcoidosis was reported by von Bartheld et al. ${ }^{26}$ Three hundred and four consecutive patients with suspected stage I/II pulmonary sarcoidosis underwent either bronchoscopy with transbronchial and endobronchial lung biopsy or endosonography (EBUS-TBNA or EUS-fine needle aspiration (FNA)). Bronchoalveolar lavage was performed in all patients. Overall the diagnostic yield to detect granulomas using endosonography $(80 \%)$ was significantly higher than using bronchoscopy with transbronchial biopsy (53\%).

\section{Mycobacterium tuberculosis}

To date, few studies have examined the role of EBUS-TBNA for the diagnosis of tuberculous intrathoracic lymphadenopathy. In a multicentre study, Navani et $a l^{27}$ examined the diagnostic sensitivity of EBUS in 156 patients with a final clinical diagnosis of 
Mycobacterium tuberculosis. EBUS-TBNA led to a diagnosis of $M$ tuberculosis in $94 \%$ of cases. Pathological findings were consistent with TB in $86 \%$ of cases and in $47 \%$ a positive culture of $M$ tuberculosis was obtained. In another series of 59 patients undergoing investigation for suspected $M$ tuberculosis, of the 41 with a final confirmed diagnosis, pathological findings were consistent with TB in $80 \%$ but in only $27 \%$ were acid-fast bacilli seen. ${ }^{28}$ Of the 37 cases put up for culture, 17 (46\%) were positive. The low positive culture rates are likely to represent the relatively low bacillary load in intrathoracic tuberculous lymph nodes and the yield obtained with EBUS in this series is similar to that reported using other modalities such as mediastinoscopy and EUS-guided biopsy. Overall, EBUS-TBNA appears to be a useful first-line investigation in patients suspected of having M tuberculosis.

\section{Investigation of suspected lymphoma}

The role of EBUS-TBNA in the diagnosis and management of lymphoma is unclear. Retrospective studies have shown varying sensitivities from $57 \%$ to $91 \%$. Kennedy et al, ${ }^{29}$ in a retrospective review of 25 patients who underwent EBUS-TBNA for intrathoracic lymphadenopathy of uncertain cause, demonstrated that 10 of the 11 patients with a final diagnosis of lymphoma were correctly identified giving a sensitivity of $90.9 \%$ and a specificity of $100 \%$. In a recent retrospective study reviewing 65 patients with a new diagnosis of lymphoproliferative disease who had undergone EBUS, Iqbal et $a l^{30}$ found the sensitivity for a definitive diagnosis of lymphoma was only $38 \%$; $22 \%$ in cases of new diagnosis and $55 \%$ for patients with recurrent disease. While a presumptive diagnosis of lymphoproliferative disease may be established by EBUS using a combination of cytopathology and flow cytometry, a surgical biopsy is often required to confirm a specific histological subtype and/or grade. This issue was highlighted in a study by Steinfort et $a l^{31}$ who used EBUS-TBNA in 55 patients with isolated mediastinal lymphadenopathy. Non-diagnostic cases underwent surgical biopsy or radiological follow-up. A final diagnosis of lymphoma was made in 16 patients. However, of these, a further surgical biopsy was required in four patients for tumour classification. In another five cases, surgical biopsy revealed lymphoma following a non-diagnostic EBUS-TBNA. Although a diagnostic sensitivity rate of $76 \%$ was reported, this becomes $57 \%$ when adjustment is made for cases that required surgical biopsy for definitive diagnosis.

More recently, a retrospective study by Moonim et $a l^{32}$ reported a sensitivity of $89 \%$ in 100 patients with mediastinal lymphoma. The patient cohort included new diagnoses and relapsed cases. Rapid on-site pathology was used to target lymph nodes with the most cellular aspirates, and a relatively high number of passes were made, mean 6.7 per lymph node for cases of Hodgkin's lymphoma. The cytopathology results were independently reviewed by the institution's haematooncology team, and further tissue sampling, largely with mediastinoscopy, was deemed necessary in 28 of 100 cases. There was 93\% concordance between paired EBUS-TBNA and histology samples. While this study does support the use of EBUS-TBNA as an initial diagnostic tool in suspected de novo or recurrent lymphoma, it should be recognised that it was undertaken by an expert group working closely with lymphoma pathologists and a lymphoma multidisciplinary team (MDT).

Although the current British Thoracic Society guidelines do not recommend the use of EBUS for diagnosis of de novo cases of lymphoma, recent reports suggest that EBUS may have an important role to play in this setting and a re-evaluation of the current guidance through carefully designed prospective studies would be timely. In the meantime, although surgical biopsy remains the initial diagnostic procedure of choice, close liaison between bronchoscopists, thoracic surgeons and the lymphoma MDT is recommended to achieve the optimal approach to diagnosis.

\section{ADEQUACY OF EBUS-TBNA ASPIRATES FOR SUBTYPING AND GENOTYPING LUNG CANCER}

The development of novel agents designed to selectively target molecular pathways in the last few years has meant that it is increasingly important for pathologists to have sufficient material for subtyping and genotyping lung cancer. At the same time, the increasing use of minimally invasive sampling techniques such as EBUS and EUS has resulted in the provision of smaller fine-needle aspirate samples. In order to address these issues, new tissue processing techniques using liquid-based thin-layer cytology and cell blocks have been developed. ${ }^{33}$ These approaches have increased diagnostic yield and provide material for immunohistochemical staining. Although there have been concerns that fine-needle aspirate cytology samples might not provide sufficient material for subtyping and genotyping, this has not proved to be the case. In a large series involving 774 patients, Navani et $a l^{34}$ showed that accurate subtyping of EBUS-TBNA samples was possible in $77 \%$ of cases and genotyping for epidermal growth factor receptor (EGFR) mutations was possible in 90\% (107 of 119 cases). In another series of 42 lung cancer patients diagnosed with EBUS-TBNA, overall success rate for EGFR mutation analysis was 95\%, KRAS mutation 90.5\% and anaplastic lymphoma kinase $90.5 \% .^{35}$ These are similar values to those that can be obtained with histological biopsies.

\section{ENDOSCOPIC ULTRASOUND}

Linear EUS is similar to EBUS although using an oesophageal approach. EUS can be used to evaluate lymph nodes lying to the left side of the trachea and in the inferior/posterior mediastinum -stations $2 \mathrm{~L}, 4 \mathrm{~L}, 7,8$ and 9. The latter two groups are not accessible from the airway. In addition, the left adrenal gland can be assessed and biopsied as can the left lobe of the liver and the coeliac lymph nodes.

\section{COMBINED EBUS AND EUS}

EBUS and EUS can be performed together under local anaesthetic and sedation. In combination, the entire mediastinum, with the exception of station 5 and 6 lymph nodes, can be assessed as well as hilar lymph node stations (10 and 11) along with the left adrenal, left lobe of liver and coeliac nodes. In practice, the dual procedures are generally well tolerated and can be performed back-to-back by an experienced team in about $50 \mathrm{~min}$.

Annema and colleagues performed the first randomised controlled trial comparing combined EBUS and EUS with surgical staging (ASTER trial). ${ }^{36}$ In the EBUS/EUS arm, cases that were negative for malignancy were referred for surgical staging, usually mediastinoscopy, prior to thoracotomy. In the endoscopic arm, the sensitivity for detection of malignancy was $85 \%$, which was not significantly different to $79 \%$ in the surgical arm. The addition of mediastinoscopy in the case of a negative EBUS/EUS increased sensitivity to $94 \%$. However, this $9 \%$ increase in sensitivity translated into the need for 11 mediastinoscopies in order to identify one patient with mediastinal nodal metastasis.

In practice, this study has shown that a combined EBUS/EUS performed by experienced operators has similar sensitivity to 
surgical staging. Although some commentators recommend that all negative EBUS/EUS examinations are referred for surgical staging prior to thoracotomy, some experienced endosonography teams only refer patients where clinical suspicion of mediastinal disease remains high.

\section{EBUS AND EUS USING THE EBUS BRONCHOSCOPE}

Some groups now perform combined EBUS and EUS using an EBUS bronchoscope. Hwangbo et $a l^{37}$ investigated 150 consecutive patients with potentially operable (suspected) non-small cell lung cancer requiring mediastinal staging. The sensitivity, negative predictive value and diagnostic accuracy of EBUS-TBNA for the detection of mediastinal metastases were 84\%, 93\% and 95\%, respectively. Performing EUS with an EBUS bronchoscope (termed EUS-B) to assess nodal stations inaccessible by EBUS improved these outcomes to $91 \%, 96 \%$ and $97 \%$, respectively. While there are advantages to performing EUS with an EBUS bronchoscope in terms of financial investment, operating costs and investigation time, there are also limitations. The EBUS scope does not permit the scanning range and ultrasound penetration depth afforded by the EUS scope, and the ultrasound quality is also inferior due to the smaller transducer. In practice, anatomical orientation within the mediastinum can be more challenging and aspiration of lymph nodes can be more difficult as the EBUS scope provides a less stable platform during fineneedle aspiration than a conventional EUS endoscope.

To assess the best approach to mediastinal staging using EBUS and EUS-B, Kang et al randomised patients to an EUS-centred approach in which EUS-B was performed initially, followed by EBUS or to an EBUS-centred approach during which EBUS was performed initially followed by EUS-B. While adding EUS-FNA to EBUS-TBNA did not significantly increase the accuracy or sensitivity of detecting mediastinal metastases, adding EBUS-TBNA to EUS-FNA increased the accuracy from $86.5 \%$ to $97.3 \%(p=0.016)$ and sensitivity from $60.0 \%$ to $92.0 \%$ $(\mathrm{p}=0.0080) .{ }^{38}$ These results suggest that commencing mediastinal staging with EBUS-TBNA and adding EUS-FNA, in selected cases, may be the most efficacious approach.

\section{COST-EFFECTIVENESS OF ENDOSONOGRAPHY}

To date, there have been few studies examining the costeffectiveness of endosonography and/or mediastinal staging. Previous work has been based on retrospective data and has used decision analysis approaches to produce models of possible outcomes and applied cost-minimisation analysis in order to determine the most economical healthcare strategy among various alternatives. ${ }^{39} 40$ The recently published 2011 National Institute for Health and Care Excellence guideline for lung cancer diagnosis and treatment includes an economic model for a number of potential diagnostic pathways. ${ }^{41}$ In the absence of empirical evidence, the model was largely based on expert opinion. Sharples et $a l^{42}$ have recently reported survival, quality of life and resource use for patients in the ASTER trial from a UK perspective. Cost-effectiveness using economic methods predominant in the UK showed that the endosonography strategy was cheaper and patients had better quality of life during staging. In recognition that health economic methodology varies substantially between different countries, Rintoul et $a l^{43}$ undertook country-specific cost-effectiveness analysis. In all three countries, the endosonography strategy had slightly higher quality-adjusted life-years over 6 months and was cheaper. Taking the clinical, quality of life and cost-effectiveness data together, the authors concluded that mediastinal staging should commence with endosonography.

\section{COMPLICATIONS}

EBUS-TBNA is regarded as a safe technique with a good safety profile. Major complications related to EBUS-TBNA are rare. For the most part, complications are minor and similar to those of standard videobronchoscopy-cough, hypoxaemia and selfterminating postprocedure pyrexia. In their meta-analysis, $\mathrm{Gu}$ et $a l^{11}$ reported only two complications in 1299 procedures $(0.15 \%)$. As the number of procedures being performed globally has risen in the last few years, case reports of more serious complications including pneumothorax, clinically significant airway injury, lung abscess, mediastinitis and haemopneumomediastinum have been published. One procedure-related death has been reported due to a postprocedure streptococcal septicaemia likely caused at intubation as a result of nasopharyngeal carriage. ${ }^{34}$ A recent study reviewing complications in 190 studies totalling 16181 cases of EUS or EBUS reported a serious adverse event rate of $0.05 \%$ for EBUS and $0.3 \%$ for EUS, although the authors state that they suspect that complications are often underreported. ${ }^{44} \mathrm{~A}$ recent prospective registry study evaluating 1317 EBUS-TBNA cases showing a complication rate of $1.44 \%$ would support this assertion. ${ }^{45}$

\section{TRAINING, IMPLEMENTATION AND SERVICE PROVISION}

Over the last 10 years, EBUS has spread rapidly among respiratory physicians and thoracic surgeons. As with many new techniques, initial publications come from a small number of groups who gain early expertise and EBUS is no exception. There is always a risk with any complex procedure requiring manual dexterity and hand-eye coordination that the 'headline' outcomes in terms of published diagnostic sensitivity and accuracy are not achieved by all and that the technique looses credibility as it is practised more widely. Therefore, some form of training requirement and ongoing competency assessment will be required.

To date, relatively little data have been published about the learning experience of EBUS-TBNA. Several groups have commented on their 'learning curve' within reports about their initial experiences of using EBUS-TBNA. ${ }^{46} 47$ The most systematic evaluation by Kemp et $a l^{48}$ undertook cumulative sum analysis on the first 100 procedures performed by five experienced bronchoscopists learning EBUS-TBNA. This showed that despite extensive bronchoscopy experience there was quite marked variation in length of time before competence in EBUS-TBNA was achieved. At present, there are no specific guidelines on training required either in terms of education or number of cases to be performed. In practice, many learners attend a dedicated course about EBUS-TBNA and visit an experienced centre to observe cases or practise on a virtual reality simulator. An EBUS Skills and Tests Assessment Tool is available. ${ }^{49}$ To date many national societies have avoided stipulating a specific number of procedures to be performed before an individual is deemed competent as such numbers are often arbitrary and rather the focus should be on monitoring an individual's performance and outcomes. However, the skill level required for fully assessing and accurately staging the mediastinum including biopsying fluorodeoxyglucose-avid sub-centimetre nodes to achieve a high negative predictive value is very different to that required for biopsying a large subcarinal lymph node mass for diagnostic purposes only. In order to achieve and maintain high levels of competence, it is likely that EBUS-TBNA will be confined to larger centres specialising in lung cancer and interventional bronchoscopy. 


\section{CONCLUSION}

Over the last decade, the advent of EBUS (and EUS) for the assessment of mediastinal disease has had a huge impact and the technique has been incorporated into many major clinical guidelines. ${ }^{13} 415051$ The challenge facing clinicians now will be to learn to apply the appropriate test or sequence of tests for each patient while ensuring that the technique is practised to uniformly high standards. While endosonographic techniques can answer many questions, surgical staging will continue to have an important role to play in certain scenarios such as the diagnosis of lymphoma, mediastinal restaging and when there is dubiety following a negative endosonographic result. Close working between endosonographers, pathologists and thoracic surgeons within the MDT will be essential to optimise the outcome for every patient.

Acknowledgements We are grateful to Professor David Baldwin and Dr Neal Navani for helpful comments on an earlier draft of the manuscript.

Contributors RCR had the original concept for the review. RA conducted the initial literature search and with RCR wrote the initial draft. BD and NRC reviewed and revised the manuscript. All authors approved the final manuscript. RCR is the guarantor of the overall content.

Funding RCR is part funded by the Cambridge Biomedical Research Centre and the Cambridge Cancer Centre.

Competing interests None.

Provenance and peer review Not commissioned; externally peer reviewed.

\section{REFERENCES}

1 Hurter T, Hanrath P. Endobronchial sonography: feasibility and preliminary results. Thorax 1992;47:565-7.

2 Herth FJ, Becker HD, Ernst A. Ultrasound-guided transbronchial needle aspiration: an experience in 242 patients. Chest 2003;123:604-7.

3 Shannon JJ, Bude RO, Orens JB, et al. Endobronchial ultrasound-guided needle aspiration of mediastinal adenopathy. AJRCCM 1996;153:1424-30.

4 Okamoto H, Watanabe K, Nagatomo A. Endobronchial ultrasonography for mediastinal and hilar lymph node metastases of lung cancer. Chest 2002;121:1498-506.

5 Steinfort DP, Khor YH, Manser RL, et al. Radial probe endobronchial ultrasound for the diagnosis of peripheral lung cancer: systematic review and meta-analysis. Eur Respir J 2011;37:902-10.

6 Krasnik M, Vilmann P, Larsen SS, et al. Preliminary experience with a new method of endoscopic transbronchial real time ultrasound guided biopsy for diagnosis of mediastinal and hilar lesions. Thorax 2003;58:1083-6.

7 Herth FJF, Krasnik M, Yasufuku K, et al. Endobronchial ultrasound-guided transbronchial needle aspiration. J Bronchology 2006;13:84-91.

8 Rintoul RC, Skwarski KM, Murchison JT, et al. Endobronchial and endoscopic ultrasound-guided real-time fine-needle aspiration for mediastinal staging. Eur Respir J 2005;25:416-21.

9 Herth FJF, Eberhardt R, Vilmann P, et al. Real-time endobronchial ultrasound guided transbronchial needle aspiration for sampling mediastinal lymph nodes. Thorax 2006;61:795-8.

10 Yasufuku K, Chiyo M, Sekine Y, et al. Real-time endobronchial ultrasound-guided transbronchial needle aspiration of mediastinal and hilar lymph nodes. Chest 2004;126:122-8.

11 Gu P, Zhao Y-Z, Jiang L-Y, et al. Endobronchial ultrasound-guided transbronchial needle aspiration for staging of lung cancer: a systematic review and meta-analysis. Eur J Cancer 2009;45:1389-96.

12 Dong X, Qiu X, Liu Q, et al. Endobronchial ultrasound-guided transbronchial needle aspiration in the mediastinal staging of non-small cell lung cancer: a meta-analysis. Ann Thorac Surg 2013;96:1502-7.

13 Silvestri GA, Gonzalez AV, Jantz MA, et al. Methods for staging non-small cell lung cancer: Diagnosis and management of lung cancer, 3rd edition: American College of Chest Physicians evidence-based clinical practice guidelines. Chest 2013;143 (Suppl 5):e211S-50S.

14 Yasufuku K, Pierre A, Darling G, et al. A prospective controlled trial of endobronchial ultrasound-guided transbronchial needle aspiration compared with mediastinoscopy for mediastinal lymph node staging of lung cancer. J Thorac Cardiovasc Surg 2011;142:1393-1400.e1.

15 Nakajima T, Yasufuku K, Fujiwara T, et al. Endobronchial ultrasound-guided transbronchial needle aspiration for the diagnosis of intrapulmonary lesions. J Thorac Oncol 2008;3:985-8.
16 Tournoy KG, Rintoul RC, van Meerbeeck JP, et al. EBUS-TBNA for the diagnosis of central parenchymal lung lesions not visible at routine bronchoscopy. Lung Cancer 2009;63:45-9.

17 Zhao H, Xie Z, Zhou ZL, et al. Diagnostic value of endobronchial ultrasound-guided transbronchial needle aspiration in intrapulmonary lesions. Chin Med I (Engl) 2013;126:4312-15.

18 De Leyn P, Stroobants S, De Wever W, et al. Prospective comparative study of integrated positron emission tomography-computed tomography scan compared with remediastinoscopy in the assessment of residual mediastinal lymph node disease after induction chemotherapy for mediastinoscopy-proven stage IIIA-N2 non-small cell lung cancer: a Leuven Lung Cancer Group Study. J Clin Oncol 2006;24:3333-9.

19 Mateu-Navarro M, Rami-Porta R, Bastus-Piulats R, et al. Remediastinoscopy after induction chemotherapy in non-small cell lung cancer. Ann Thorac Surg 2000;70:391-5.

20 Marra A, Hillejan L, Fechner S, et al. Remediastinoscopy in restaging of lung cancer after induction therapy. I Thorac Cardiovasc Surg 2008;135:843-9.

21 Herth FJ, Annema JT, Eberhardt R, et al. Endobronchial ultrasound with transbronchial needle aspiration for restaging the mediastinum in lung cancer. J Clin Oncol 2008;26:3346-50

22 Szlubowski A, Zielinski M, Soja J, et al. Accurate and safe mediastinal restaging by combined endobronchial and endoscopic ultrasound-guided needle aspiration performed by single ultrasound bronchoscope. Eur I Cardiothorac Surg 2014;46:262-6.

23 Navani N, Nankivell M, Woolhouse I, et al. Endobronchial ultrasound-guided transbronchial needle aspiration for the diagnosis of intrathoracic lymphadenopathy in patients with extrathoracic malignancy: a multicenter study. J Thorac Oncol 2011;6:1505-9.

24 Tournoy KG, Govaerts E, Malfait T, et al. Endobronchial ultrasound-guided transbronchial needle biopsy for M1 staging of extrathoracic malignancies. Ann Oncol 2011;22:127-31.

25 Agarwal R, Srinivasan A, Aggarwal AN, et al. Efficacy and safety of convex probe EBUS-TBNA in sarcoidosis: a systematic review and meta-analysis. Respir Med 2012;106:883-92.

26 von Bartheld MB, Dekkers OM, Szlubowski A, et al. Endosonography vs conventional bronchoscopy for the diagnosis of sarcoidosis: the GRANULOMA randomized clinical trial. JAMA 2013;309:2457-64.

27 Navani N, Molyneaux PL, Breen RA, et al. Utility of endobronchial ultrasound-guided transbronchial needle aspiration in patients with tuberculous intrathoracic lymphadenopathy: a multicentre study. Thorax 2011;66:889-93.

28 Sun J, Teng J, Yang H, et al. Endobronchial ultrasound-guided transbronchial needle aspiration in diagnosing intrathoracic tuberculosis. Ann Thorac Surg 2013;96:2021-7.

29 Kennedy MP, Jimenez CA, Bruzzi JF, et al. Endobronchial ultrasound-guided transbronchial needle aspiration in the diagnosis of lymphoma. Thorax 2008;63:360-5.

30 lqbal S, DePew ZS, Kurtin PJ, et al. Endobronchial ultrasound and lymphoproliferative disorders: a retrospective study. Ann Thorac Surg 2012:94:1830-4.

31 Steinfort DP, Conron M, Tsui A, et al. Endobronchial ultrasound-guided transbronchial needle aspiration for the evaluation of suspected lymphoma. J Thorac Oncol 2010;5:804-9.

32 Moonim MT, Breen R, Fields PA, et al. Diagnosis and subtyping of de novo and relapsed mediastinal lymphomas by endobronchial ultrasound needle aspiration. AJRCCM 2013;188:1216-23.

33 Wallace WA, Rassl DM. Accuracy of cell typing in nonsmall cell lung cancer by EBUS/EUS-FNA cytological samples. Eur Respir J 2011;38:911-17.

34 Navani N, Brown JM, Nankivell M, et al. Suitability of endobronchia ultrasound-guided transbronchial needle aspiration specimens for subtyping and genotyping of non-small cell lung cancer: a multicentre study of 774 patients. AJRCCM 2012;185:1316-22.

35 Folch E, Yamaguchi N, VanderLaan PA, et al. Adequacy of lymph node transbronchial needle aspirates using convex probe endobronchial ultrasound for multiple tumor genotyping techniques in non-small-cell lung cancer. J Thorac Oncol 2013;8:1438-44.

36 Annema JT, van Meerbeeck PJ, Rintoul RC, et al. Mediastinoscopy vs endosonography for mediastinal nodal staging of lung cancer: a randomized trial. JAMA 2010;304:2245-52.

37 Hwangbo B, Lee GK, Lee HS, et al. Transbronchial and transesophageal fine-needle aspiration using an ultrasound bronchoscope in mediastinal staging of potentially operable lung cancer. Chest 2010;138:795-802.

38 Kang HJ, Hwangbo B, Lee GK, et al. EBUS-centred versus EUS-centred mediastinal staging in lung cancer: a randomised controlled trial. Thorax 2014;69:261-8.

39 Steinfort DP, Liew D, Conron M, et al. Cost-benefit of minimally invasive staging of non-small cell lung cancer: a decision tree sensitivity analysis. $J$ Thorac Oncol 2010;5:1564-70

40 Harewood GC, Pascual J, Raimondo M, et al. Economic analysis of combined endoscopic and endobronchial ultrasound in the evaluation of patients with suspected non-small cell lung cancer. Lung Cancer 2010;67:366-71. 
41 National Institute for Health and Care Excellence. Lung cancer. The diagnosis and treatment of lung cancer. 27 April 2011. http://www.nice.org.uk/nicemedia/live/ 13465/54202/54202.pdf (accessed 4 Apr 2014).

42 Sharples LD, Jackson C, Wheaton E, et al. Clinical effectiveness and cost effectiveness of endobronchial and endoscopic ultrasound relative to surgical staging in potentially resectable lung cancer: results from the ASTER randomised controlled trial. Health Technol Assess

2012;16:175. iii-iv.

43 Rintoul RC, Glover MJ, Jackson C, et al. Cost effectiveness of endosonography versus surgical staging in potentially resectable lung cancer: a health economics analysis of the ASTER trial from a European perspective. Thorax 2014;69:679-81.

44 von Bartheld MB, van Breda A, Annema JT. Complication rate of endosonography (endobronchial and endoscopic ultrasound): a systematic review. Respiration 2014;87:343-51.

45 Eapen GA, Shah AM, Lei $X$, et al. Complications, consequences, and practice patterns of endobronchial ultrasound-guided transbronchial needle aspiration: results of the AQulRE registry. Chest 2013;143:1044-53.
46 Steinfort DP, Hew MJ, Irving LB. Bronchoscopic evaluation of the mediastinum using endobronchial ultrasound: a description of the first 216 cases carried out at an Australian tertiary hospital. Intern Med J 2011;41:815-24.

47 Groth SS, Whitson BA, D'Cunha J, et al. Endobronchial ultrasound-guided fine-needle aspiration of mediastinal lymph nodes: a single institution's early learning curve. Ann Thorac Surg 2008;86:1104-9.

48 Kemp SV, El Batrawy SH, Harrison RN, et al. Learning curves for endobronchial ultrasound using cusum analysis. Thorax 2010;65:534-8.

49 Davoudi M, Colt HG, Osann KE, et al. Endobronchial ultrasound skills and tasks assessment tool: assessing the validity evidence for a test of endobronchial ultrasound-guided transbronchial needle aspiration operator skill. Am J Respir Crit Care Med 2012;186:773-9.

50 De Leyn P, Dooms C, Kuzdzal J, et al. Revised ESTS guidelines for preoperative mediastinal lymph node staging for non-small-cell lung cancer. Eur J Cardiothorac Surg 2014;45:787-98.

51 Du Rand IA, Barber PV, Goldring J, et al. British Thoracic Society guideline for advanced diagnostic and therapeutic flexible bronchoscopy in adults. Thorax 2011;66:iii1-21. 